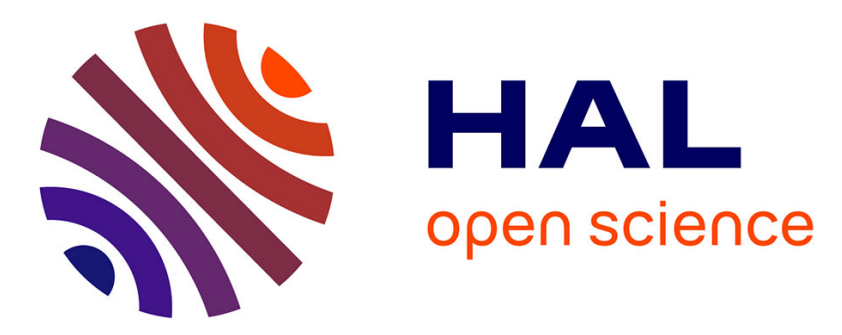

\title{
Destructive interferences between diffuse scatterings due to disorder and displacive modulation (X-ray "white line" effect)
}

\author{
S. Ravy, Jean Pouget, R. Comes
}

\section{- To cite this version:}

S. Ravy, Jean Pouget, R. Comes. Destructive interferences between diffuse scatterings due to disorder and displacive modulation (X-ray "white line" effect). Journal de Physique I, 1992, 2 (6), pp.11731190. 10.1051/jp1:1992203 . jpa-00246596

\section{HAL Id: jpa-00246596 https://hal.science/jpa-00246596}

Submitted on 1 Jan 1992

HAL is a multi-disciplinary open access archive for the deposit and dissemination of scientific research documents, whether they are published or not. The documents may come from teaching and research institutions in France or abroad, or from public or private research centers.
L'archive ouverte pluridisciplinaire HAL, est destinée au dépôt et à la diffusion de documents scientifiques de niveau recherche, publiés ou non, émanant des établissements d'enseignement et de recherche français ou étrangers, des laboratoires publics ou privés. 


\title{
Destructive interferences between diffuse scatterings due to disorder and displacive modulation (X-ray « white line » effect)
}

\author{
S. Ravy, J. P. Pouget and R. Comes (*) \\ Laboratoire de Physique des Solides (CNRS-URA 2), Bât. 510, Université Paris-Sud, 91405 \\ Orsay Cedex, France
}

(Received 15 October 1991, accepted 16 December 1991)

\begin{abstract}
Résumé. - Dans des solutions solides de sels à transfert de charge de la famille TTF-TCNQ un effet de diffraction original est observé. Les figures de diffraction de tels matériaux font apparaître de larges diffusions diffuses sur lesquelles on observe de fines lignes diffuses «blanches » en $\mathbf{Q}+2 \mathbf{k}_{\mathrm{f}}$, alors qu'en $\mathbf{Q}-\mathbf{2} \mathbf{k}_{\mathrm{f}}$ on retrouve l'aspect habituel des lignes noires. Nous proposons une interprétation simple de cette asymétrie d'intensité $+2 \mathbf{k}_{\mathrm{f}} f-2 \mathbf{k}_{\mathrm{f}}$ en couplant en quadrature une onde de déplacement et une onde de composition de même vecteur d'onde. La cohérence entre le désordre de substitution et le déplacement étant à l'origine de l'effet, nous effectuons ensuite un calcul de l'intensité diffusée au premier ordre en déplacement pour un modèle d'alliage unidimensionnel dans lequel toutes les impuretés accrochent une distorsion de réseau à $2 \mathbf{k}_{\mathrm{f}}$, avec la même phase. Ces résultats nous permettent de mettre en évidence les trois paramètres pertinents du problème : la différence entre les facteurs de structure des entités substituées, la phase de l'onde accrochée par rapport à l'impureté et sa polarisation. Nous proposons que les lignes blanches observées expérimentalement dans (HMTTF) $)_{0,05}$ (HMTSF) $)_{0,95}$-TCNQ résultent des distorsions de réseau couplées aux oscillations de charge induites par des impuretés sur les chaînes donneuses.
\end{abstract}

\begin{abstract}
In the solid solutions of charge transfer salts of the TTF-TCNQ family, a new diffraction effect is observed. The X-ray patterns of such materials show a broad diffuse scattering on which fine "white " diffuse lines at $\mathbf{Q}+2 \mathbf{k}_{\mathrm{f}}$ are observed, whereas at $\mathbf{Q}-2 \mathbf{k}_{\mathrm{f}}$ the diffuse scattering has the usual aspect. We propose a simple interpretation of this $+2 \mathbf{k}_{f}-2 \mathbf{k}_{f}$ asymmetry of intensity by a coupling in quadrature between a displacement wave and a composition wave of same wave vector. As the effect originates from a coherence between substitutional disorder and the displacement waves, we perform a calculation of the diffracted intensity to first order in displacement, for a one-dimensional alloy in which all the impurities pin a lattice distortion of wave vector $2 \mathbf{k}_{\mathrm{f}}$, with the same phase. We emphasize the three relevant parameters of the calculations : the difference of structure factors of the substituted entities, the phase of the displacement wave with respect to the impurity and its polarization. We then propose that the white lines experimentally observed in (HMTTF) 0.05 (HMTSF) 095 -TCNQ, originate from the $2 \mathbf{k}_{\mathrm{f}}$ distortions induced by charge oscillations around the substituted HMTTF molecules on the donor stacks.
\end{abstract}

(*) Present Address : LURE, Bât. 209 D, Université Paris-Sud, 91405 Orsay Cedex, France. 


\section{Introduction.}

The organic charge transfer salts of the TTF-TCNQ family have been extensively studied in the past [1]. They are composed of segregated one-dimensional (1D) chains of donors (TTF, HMTTF or HMTSF) and acceptors (TCNQ), on which electrons are delocalized. These 1D conductors are known to be unstable with respect to the formation of charge density wave at twice the Fermi wave vector $\left(2 \mathbf{k}_{\mathrm{f}}\right)$. Below $T_{\mathrm{p}}$, the Peierls transition temperature, a $2 \mathbf{k}_{\mathrm{f}}$ periodic lattice distortion is stabilized and a gap is opened at the Fermi level, the gain of electronic energy being greater than the loss of elastic energy. In a large temperature range above $T_{\mathrm{p}}$, a regime of $1 \mathrm{D}$ pretransitional fluctuations is observed. $\mathrm{X}$-ray diffuse scattering is a powerful technique to study this $1 \mathrm{D}$ regime [2]. The lattice distortion coupled of the electron gas gives rise to diffuse planes located symmetrically at $\pm 2 \mathbf{k}_{\mathbf{f}}$ of the layers of Bragg spots perpendicular to the 1D direction. By intersection to the Ewald sphere these planes yield diffuse lines on the X-ray pattern. A thorough study of these lines gives direct information on the in-chain correlation length $\xi$ of the lattice fluctuations and on the susceptibility of the coupled electron-phonon system.

The effects of disorder, especially singular in chain-like materials, were investigated by studying the solid solutions (TTF) $)_{x}$ (TSF) $)_{1-x}$ TCNQ and (HMTTF $)_{x}(\text { HMTSF })_{1-x}$ TCNQ, were selenium- and sulfur-based donor molecules are alloyed. An X-ray pattern obtained for

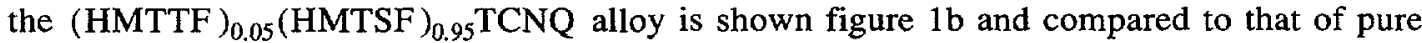
HMTSF-TCNQ (Fig. 1a). In figure $1 \mathrm{~b}$ a very striking asymmetry in the intensities of the $\pm 2 \mathbf{k}_{\mathrm{f}}$ diffuse lines is observed. At $+2 \mathbf{k}_{\mathrm{f}}$, the lines have an intensity minimum visible at the expense of the background (white arrows) and at $-2 \mathbf{k}_{\mathrm{f}}$ an intensity maxima (black arrows). It was suggested [3] that the $2 \mathbf{k}_{\mathrm{f}}$ white lines originate from a substraction of a diffuse intensity term, due to lattice fluctuations, from the so-called «monotonic " Laue scattering due to the HMTTF/HMTSF disorder. Such negative and positive interferences were assumed to result from a phase coherence between the position of the impurities and the lattice distorsions. This paper presents calculations which confirm these ideas. In the first section, we recall that in a system where there is a $90^{\circ}$ out-of-phase coupling between a displacement wave and a composition wave of same wave vector $\mathbf{q}$, an asymmetry of the intensity of the $\pm q$ satellite reflections located on each side of the Bragg reflections is expected. This asymmetry is a key to understanding the white line effect. An exact calculation of first-order

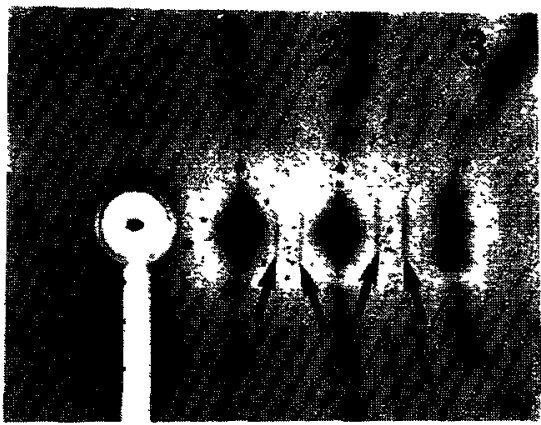

a)

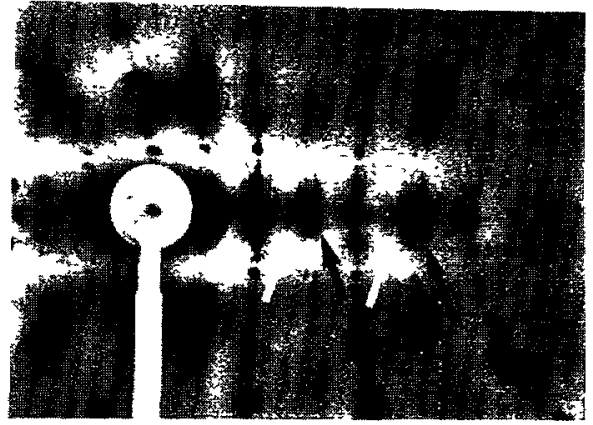

b)

Fig. 1. - a) X-ray pattern from HMTSF-TCNQ at $25 \mathrm{~K}$ showing $2 \mathbf{k}_{\mathrm{f}}$ diffuse lines (black arrows). b) Xray pattern from the solid solution (HMTTF $)_{0.05}(\mathrm{HMTSF})_{0}{ }_{95} \mathrm{TCNQ}$ at $20 \mathrm{~K}$ showing the white diffuse lines at $+2 k_{\mathrm{f}}$ (white arrows) and the black diffuse lines at $-2 \mathbf{k}_{\mathrm{f}}$ (black arrows) for $\ell>0$. Both $X$-rays patterns have been taken with about the same crystal orientation, with the $c$ axis horizontal and the $b$ axis vertical. The wavelengh used was $\lambda_{\mathrm{Cu} \mathrm{K \alpha}}=1.542 \AA$. 
terms in displacement, which are responsible for the effect, is performed in section 2 for a model system in which each impurity pins a displacement wave on a coherence length $\xi$, with the same relative phase. The relevant parameters are then discussed and experimental evidence is briefly shown.

\section{The origin of the white lines.}

2.1 THE ASYMMETRY OF SATELLITE REFLECTIONS. - In order to clearly emphasize the physical origin of this effect, it is useful to reconsider a simple calculation first performed by Guinier [4]. Let us consider a one-dimensional lattice of period $a$, composed of $N$ atoms, the atomic scattering factor $f_{n}$ of which varies from site to site as a sine function

$$
f_{n}=f[1+\eta \sin (2 \pi q n a+\psi)],
$$

where $q$ is the wave vector of the modulation, $\eta$ its amplitude and $\psi$ its phase. This expression can, for example, account for the spatial dependence of the mean structure factor of an atomic occupation wave in a binary alloy. More generally it can represent the generic term of the Fourier transform of any composition modulation.

Now, let us consider a sinusoidal displacement wave as follows

$$
x_{n}=n a+u \sin (2 \pi q n a+\varphi),
$$

where $u$ is the amplitude of the displacement. This modulation has the same wave vector $q$ as the composition modulation but a different phase, $\varphi$. We define $\Phi$ as the phase difference between the two modulations :

$$
\Phi \equiv \varphi-\psi
$$

The diffracted amplitude $A(s)$ is given by

$$
A(s)=\sum_{n} f_{n} \exp \left(-2 i \pi s x_{n}\right)
$$

where the index $n$ runs through the $N$ sites and $s$ represents the scattering wave vector. We have then to calculate the expression

$$
\sum_{n} f(1+\eta \sin (2 \pi q n a+\psi)) \exp (-2 i \pi s(n a+u \sin (2 \pi q n a+\varphi))) .
$$

The exact calculation is performed in appendix $\mathrm{A}$, but for the sake of simplicity the values of the peak intensities up to the second order in $u$ are indicated in tables I and II, with the usual approximation $2 \pi s u \ll 1$. Figure 2 summarizes the following situations :

- In the case of a pure composition wave, the reciprocal space consists of nodes at $s=Q=h / a$ ( $h$ integer) and of pairs of satellites located at $s=Q \pm q$, the intensities of which are proportionnal to $\eta^{2} / 4$. Characteristic of such a modulation is the fact that these intensities are independent of the scattering angle, when the $s$-variations of the scattering factor $f$ are ignored.

- In the case of a pure displacement wave, pairs of satellites appear at $s=Q \pm m q$ ( $m$ integer), with intensities proportional to $\left(J_{m}(2 \pi(Q \pm m q) u)\right)^{2}$, where $J_{m}$ is the Bessel function of $m$-th order. Generally, the amplitudes of distortion are small and the high order $(|m|>1)$ satellite intensities are very small. Contrary to the previous case, the intensity of the satellite reflections increases with the scattering angle. 
Table I

\begin{tabular}{|c|c|c|}
\cline { 2 - 3 } \multicolumn{1}{c|}{} & Pure composition wave & Pure displacement wave \\
\hline $\mathrm{s}=Q$ & $N^{2} f^{2}$ & $N^{2} f^{2}\left(1-(\pi Q u)^{2}\right)$ \\
\hline $\mathrm{s}=Q+q$ & $N^{2} f^{2} \eta^{2} / 4$ & $N^{2} f^{2}\left(\pi(Q+q) u^{2}\right)$ \\
\hline $\mathrm{s}=Q-q$ & $N^{2} f^{2} \eta^{2} / 4$ & $N^{2} f^{2}\left(\pi(Q-q) u^{2}\right)$ \\
\hline
\end{tabular}

Table II

\begin{tabular}{|c|c|}
\hline & Coupled composition and displacement waves \\
\hline $\mathrm{s}=Q$ & $-N^{2} f^{2}(\pi Q u)^{2}\left(\eta^{2} \cos ^{2} \Phi-2\right)$ \\
\hline $\mathrm{s}=Q+q$ & $\begin{array}{r}N^{2} f^{2} \eta^{2} / 4+N^{2} f^{2} \eta \pi(Q+q) u \sin \Phi+N^{2} f^{2}(\pi(Q+q) u)^{2} \\
\left\{\left(1-\eta^{2}(2+\cos 2 \Phi)\right) / 4\right\}\end{array}$ \\
\hline $\mathrm{s}=Q-q$ & 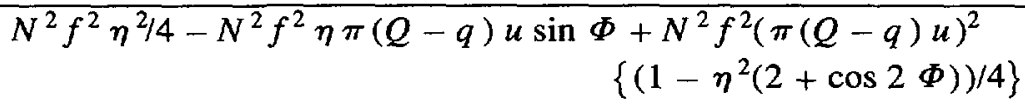 \\
\hline
\end{tabular}

(a)

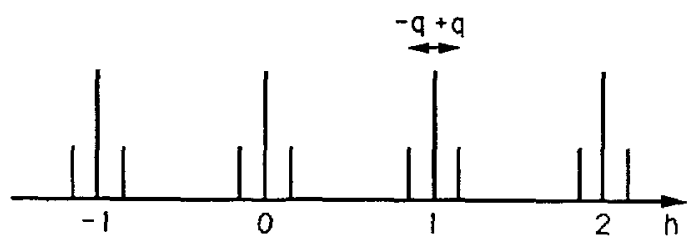

(b)

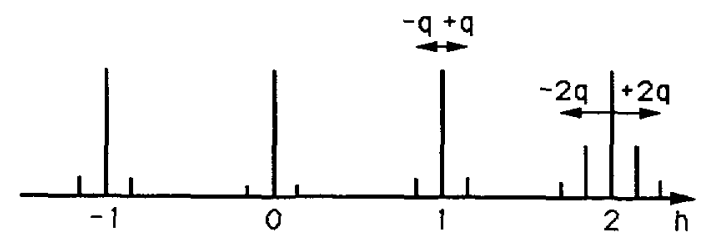

(c)

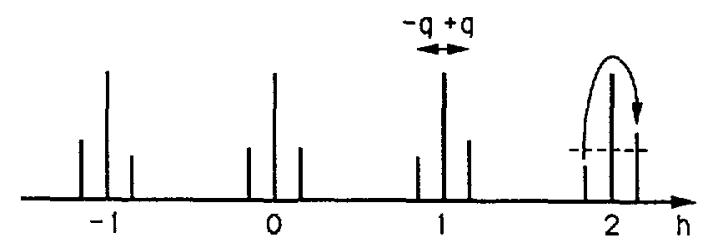

Fig. 2. - a) Schematic representation of the reciprocal lattice of a one-dimensional system with a composition wave of wave vector $\mathbf{q}(h$ integer). b) Same representation for a displacive modulation of wave vector $q$. The intensity of the $q$-satellite peaks increases and high order peaks become visible for large $h$ values. c) Same representation for a coupling between composition and displacive modulations of same wave vector with $\eta(Q+q) u \sin \varphi$ positive. For $h \neq 0$, some intensity is transferred from one side of a Bragg reflection to the other side, inducing an asymmetry $+\mathbf{q},-\mathbf{q}$ on the satellite intensity. 
- When both modulations are present, a $\eta u$ bilinear term appears in the expression of the first order satellite intensity. The sign of this term is opposite for the two $Q \pm q$ satellites and, provided that $\sin \Phi \neq 0$, the intensity becomes asymmetric with respect to the nodes of the average lattice. The $N^{2} f^{2} \eta \pi(Q+q) u \sin \Phi$ intensity is transferred from one satellite reflection to the other one (Fig. 2). As first emphasized by Guinier [4] this phenomena is characteristic of a correlation between a composition and a displacement wave.

Let us discuss more precisely the importance of the bilinear term $\eta \pi(Q \pm q) u \sin \Phi$. If $s u \ll \eta$ and $\sin \Phi \neq 0$ the classical second order term is neglected and the intensities of the satellites are given by

$$
I(Q \pm q)=N^{2} f^{2} \eta^{2} / 4 \pm N^{2} f^{2} \eta \pi(Q \pm q) u \sin \Phi .
$$

These intensities crucially depend on the sign of the bilinear term which itself depends on $\eta, \pi(Q \pm q) u$ and $\sin \Phi$. The influence of the two first terms will be discussed in the following but the role of the latter term is quite straightforward.

If the modulated waves are in phase or $180^{\circ}$ out-of-phase, the bilinear term vanishes. The satellites intensities are given by the second order term and no asymmetry appears. If $\Phi \neq 0$ or $\pi$, the satellites have not the same intensity except around the origin of the reciprocal space $Q=0$ and the relative difference of intensity is maximum when $\Phi= \pm \pi / 2$. This latter situation is of physical importance. For example it corresponds to the so-called size effect [5] in alloys where the $s$ variation of the diffuse scattering arises from lattice distortion resulting from the different size of the constituting atoms. We shall see that the white line effect has a similar origin.

2.2 DISTORTION WAVE PINNED TO AN IMPURITY. - Now, let us consider a lattice of $N+1$ atoms $\mathrm{B}$ with a foreign atom $\mathrm{A}$ at the $n=0$ position. The atomic positions are given by

$$
x_{n}=n a+u \sin (2 \pi q n a+\varphi),
$$

as shown in figure 3 .

This very simple model represents a displacement modulation pinned to the impurity A.

The scattering factors of such a lattice can be written as

$$
f_{n}=f_{\mathrm{B}}+\left(f_{\mathrm{A}}-f_{\mathrm{B}}\right) \delta_{n},
$$

where $f_{\mathrm{B}}$ and $f_{\mathrm{A}}$ are the scattering factor of the $\mathrm{B}$ and $\mathrm{A}$ atoms respectively. The $\delta_{n}$ function, defined by $\delta_{n}=1$ if $n=0$ and $\delta_{n}=0$ elsewhere, can be developed in a Fourier series, which gives

$$
f_{n}=f_{\mathrm{B}}+\frac{1}{N+1}\left(f_{\mathrm{A}}-f_{\mathrm{B}}\right) \sum_{q^{\prime}} \exp \left(2 i \pi q^{\prime} n a\right) \quad q^{\prime}=\frac{m}{a(N+1)}, \quad m=-N / 2, ., N / 2
$$

or,

$$
\begin{gathered}
f_{n}=\frac{2}{N+1} \sum_{q^{\prime}}\left(f_{\mathrm{B}}-f_{\mathrm{A}} /(N+2)\right)\left\{1+\frac{\left(f_{\mathrm{A}}-f_{\mathrm{B}}\right)}{f_{\mathrm{B}}-f_{\mathrm{A}} /(N+2)} \sin \left(2 \pi q^{\prime} n a+\pi / 2\right)\right\} \\
q^{\prime}=\frac{m}{a(N+1)}, \quad m=0, \ldots, N / 2
\end{gathered}
$$

$f_{n}$ given by (10) is a sum of functions defined in (1) with

$$
f=2 \frac{\left(f_{\mathrm{B}}-f_{\mathrm{A}} /(N+2)\right)}{(N+1)}, \quad \eta=\frac{\left(f_{\mathrm{A}}-f_{\mathrm{B}}\right)}{\left(f_{\mathrm{B}}-f_{\mathrm{A}} /(N+2)\right)} \quad \text { and } \quad \psi=\pi / 2 .
$$



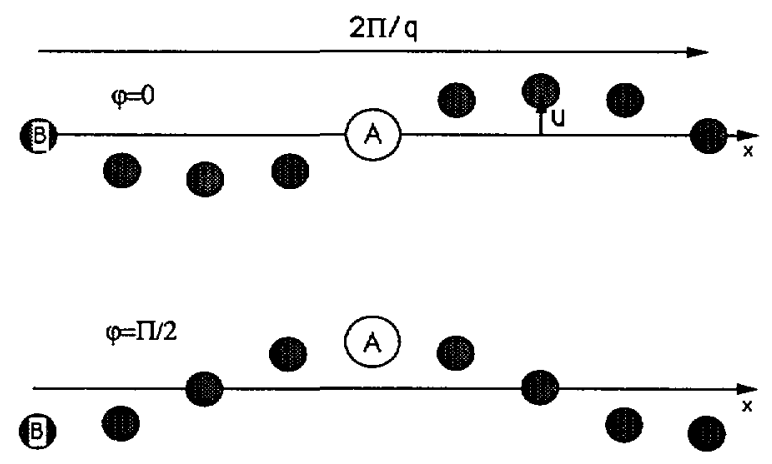

Fig. 3. - Atomic displacements for a transverse lattice distortion of wave vector $\mathbf{q}$, pinned to an impurity $A$ with relative phases $\varphi=0$ (top) and $\varphi=\pi / 2$ (bottom).

For all the non zero values of $q^{\prime}$, this variation of the scattering factor will give a set of pairs of satellites around each node. For a sufficiently high value of $N$, this set will appear as a continuous background of intensity

$$
I_{\mathrm{B}}=\left(f_{\mathrm{A}}-f_{\mathrm{B}}\right)^{2},
$$

which is just the so-called «monotonic » Laue diffuse scattering.

The bilinear terms $f^{2} \eta \pi\left(Q \pm q^{\prime}\right) u \sin (\varphi-\pi / 2)$ are non zero only when $q^{\prime}=q$, i.e. when the two modulation waves do interfere. Their values are

$$
I_{\mathrm{S}}=-4 f_{\mathrm{B}}\left(f_{\mathrm{A}}-f_{\mathrm{B}}\right) \pi(Q \pm q) u \cos \varphi \text {. }
$$

Assuming that $\left(f_{\mathrm{A}}-f_{\mathrm{B}}\right) / f_{\mathrm{B}} \gg(\pi(Q \pm q) u)$ and $\cos \varphi \neq 0$, the intensities at the $Q \pm q$ positions are

$$
\begin{aligned}
& I(Q+q)=\left\{\left(f_{\mathrm{A}}-f_{\mathrm{B}}\right)^{2}-4 f_{\mathrm{B}}\left(f_{\mathrm{A}}-f_{\mathrm{B}}\right) \pi(Q+q) u \cos \varphi\right\} \\
& I(Q-q)=\left\{\left(f_{\mathrm{A}}-f_{\mathrm{B}}\right)^{2}+4 f_{\mathrm{B}}\left(f_{\mathrm{A}}-f_{\mathrm{B}}\right) \pi(Q-q) u \cos \varphi\right\} .
\end{aligned}
$$

When $u=0$, the reciprocal space of this lattice consists of nodes at $Q=h / a$ and of a Laue «monotonic » background due to the impurity. When a displacive modulation is added, an asymmetry of the satellite intensity is observed around each node. If $I_{S}>0$, the satellite intensity at the $Q+q(Q-q)$ position is greater (weaker) than that of the Laue scattering background. Thus $Q-q$ «negative» satellite reflections will appear on the diffraction pattern (Fig. 4). The position of this negative satellite reflections (i.e. white line) with respect to the main Bragg spots depends on the phase $\varphi$ of the lattice distortion and of the scattering factors of the atoms alloyed. Let us remark that our calculation satisfies the Friedel rule because expressions (13) and (14) are interchanged by inversion symmetry $(Q \rightarrow-Q)$ around the origin of the reciprocal space.

\section{Calculation of the X-ray diffuse scattering intensity.}

3.1 FoRMUlATION OF THE PROBLEM. - Let us now consider an alloyed chain composed of two species $\mathrm{A}$ and $\mathrm{B}$ (atoms or molecules) of concentration $x_{\mathrm{A}}$ and $x_{\mathrm{B}}$, with structure factor :

$$
F_{\mathrm{A}(\mathrm{B})}=\sum_{j} f_{J} \exp \left(-2 i \pi \mathbf{r}_{j} \cdot \mathbf{s}\right),
$$



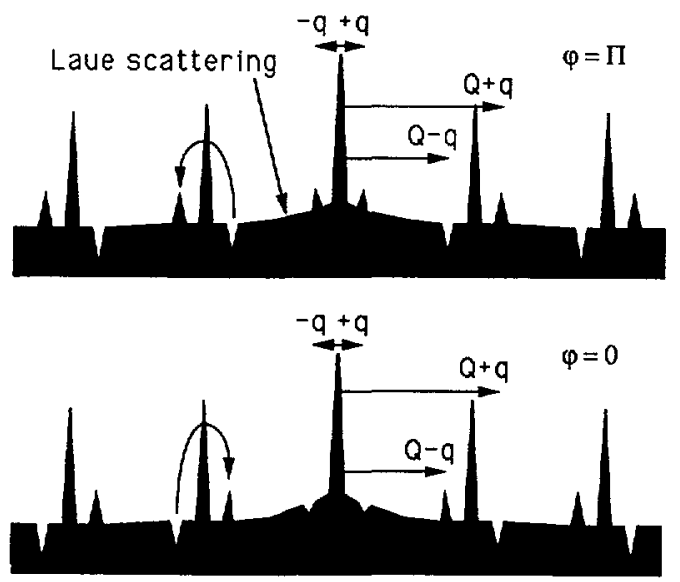

Fig. 4. - Schematic representation of the intensity scattered by a chain in which an impurity pins a lattice distortion of wave vector $q$. In the case where $f_{\mathrm{A}}>f_{\mathrm{B}}$, two extrem situations corresponding to the relative phases $\varphi=\pi$ and $\varphi=0$ are shown on top and bottom respectively.

where $f_{J}$ is the scattering factor, assumed to be real, of the $j$-th atom of the A (B) unit of position $\mathbf{r}_{\jmath}$. For the sake of simplicity, we shall assume that the structure is centrosymmetric and that the molecules themselves are centrosymmetric in order to assure that the structure factors are real quantities.

The model system studied is schematically represented in figure 5 . We assume that the $\mathrm{A}$ atoms are the impurities and that around each impurity, located at position $k \mathbf{a}$, a displacement wave is pinned. It is characterized by its wave vector $q$ and its correlation length $\xi$. The displacement of the $n$-th atom due to the $k$-th impurity is written

$$
\mathbf{u}_{n}^{k}=\sum_{\mathbf{q}^{\prime}} \mathbf{u}_{\mathbf{q}^{\prime}} \sin \left(2 \pi \mathbf{q}^{\prime}(n-k) \mathbf{a}+\varphi\right)
$$

where $\mathbf{q}^{\prime}$ belongs to the first Brillouin zone (B.Z.) and $\mathbf{u}_{\mathbf{q}}$ is a function of $\mathbf{q}^{\prime}$ centred around $\mathbf{q}$. Moreover, we shall assume that $2 \pi|\mathbf{q}| \xi \gg 1$ which ensures that

$$
\sum_{n} \mathbf{u}_{n}^{k}=\mathbf{u}_{\mathbf{q}^{\prime}=\mathbf{0}}=0
$$

It is also useful to define the $\sigma_{n}^{\mathrm{A}}$ and $\sigma_{n}^{\mathrm{B}}$ variables by $\sigma_{n}^{\mathrm{A}}=+1, \sigma_{n}^{\mathrm{B}}=0$ if the site $n$ is occupied by the molecule $\mathrm{A}$, and $\sigma_{n}^{\mathrm{A}}=0, \sigma_{n}^{\mathrm{B}}=+1$ if there is a $\mathrm{B}$ molecule at $n$.

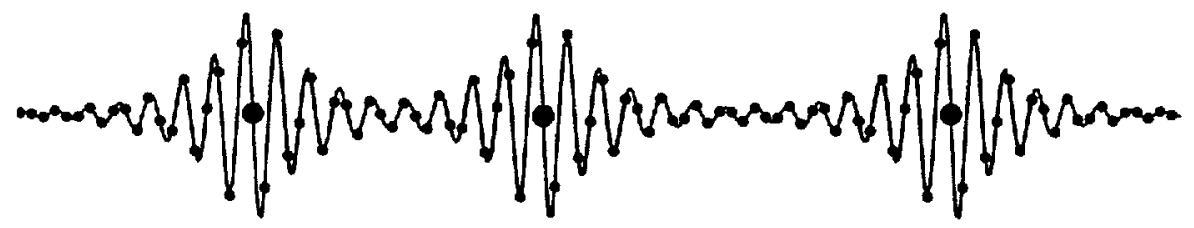

Fig. 5. - One-dimensional model used for the calculation of the diffuse intensity. All the impurities A pin displacive modulations of wave vector $q$ and spatial extension $\xi$. A and B species are represented by large and small dots respectively. 
Following these definitions the displacement of a molecule located at na is

$$
\mathbf{u}_{n}=\sum_{k} \sigma_{k}^{\mathrm{A}} \mathbf{u}_{n}^{k}
$$

and its structure factor

$$
F_{n}^{\prime}=F_{n} \exp \left(-2 i \pi \mathbf{s} \cdot \mathbf{u}_{n}\right)=\left(\sigma_{n}^{\mathrm{A}} F_{\mathrm{A}}+\sigma_{n}^{\mathrm{B}} F_{\mathrm{B}}\right) \exp \left(-2 i \pi \mathbf{s} \cdot \mathbf{u}_{n}\right),
$$

where in (18) the summation is performed over all the impurity sites $k$.

3.2 Calculation of THE DifFRACTED InTENSITY. - In the kinematic approximation (see for example Ref. [4]) the total diffracted intensity is given by

$$
I(\mathbf{s})=N \frac{|\Sigma(\mathbf{s})|^{2}}{V} * Y(\mathbf{s}),
$$

where $V$ is the volume of the crystal, $N$ is the total number of unit cells, $\Sigma(\mathbf{s})$ is the Fourier transform of the form factor $v(\mathbf{r})$ for the crystal $(v(\mathbf{r})=1$ inside the crystal and $v(\mathbf{r})=0$ outside $)$ and $Y(\mathbf{s})$ is

$$
Y(\mathbf{s})=\sum_{m}\left\langle F_{n}^{\prime} F_{n+m}^{\prime *}\right\rangle_{n} \exp (2 i \pi \mathbf{s} \cdot m \mathbf{a})
$$

$\left\langle F_{n}^{\prime} F_{n+m}^{\prime *}\right\rangle_{n}$ is the average of $F_{n}^{\prime} F_{n+m}^{\prime *}$ over all the sites of the crystal, that we suppose to be homogeneous. This total intensity contains two contributions, the sharp Bragg reflection term due to the periodicity of the average lattice,

$$
Y_{\mathrm{Bragg}}(\mathrm{s})=\sum_{m}|F|^{2} \exp (2 i \pi \mathbf{s} \cdot m \mathbf{a}),
$$

and the diffuse scattering term due to deviations from perfect order

$$
Y_{\mathrm{D} \text { Iff }}(\mathbf{s})=\sum_{m}\left\langle\left(F-F_{n}^{\prime}\right)\left(F^{*}-F_{n+m}^{\prime *}\right)\right\rangle_{n} \exp (2 i \pi \mathbf{s} \cdot m \mathbf{a}),
$$

where $F=\left\langle F_{n}^{\prime}\right\rangle_{n}$ is the average atomic (molecular) structure factor.

The calculation of the average in (23) is difficult because the correlation of structure factors of two species not only depends on their nature and their position but also on the position of all the impurities in the crystal. As first shown by Cowley [6], such an average contains highorder correlations terms.

Up to first order in displacement, we can write

$$
F=\left\langle F_{n}\right\rangle_{n}-2 i \pi\left\langle F_{n} \mathbf{u}_{n}\right\rangle_{n},
$$

and

$$
\begin{aligned}
\left\langle\left(F-F_{n}^{\prime}\right)\left(F^{*}-F_{n+m}^{\prime *}\right)\right\rangle_{n} & =\left\langle\left(F-F_{n}\right)\left(F^{*}-F_{n+m}^{*}\right)\right\rangle_{n}+ \\
+ & 2 i \pi \mathbf{s} .\left\langle F_{n}\left(F^{*}-F_{n+m}^{*}\right) \mathbf{u}_{n}-F_{n+m}^{*}\left(F-F_{n}\right) \mathbf{u}_{n+m}\right\rangle_{n}
\end{aligned}
$$

In order to calculate these terms, we have to introduce pair and triplet correlation coefficients. Let us define the two-sites probabilities $P_{\mathrm{AB}}(m)$ (resp. $P_{\mathrm{BA}}(m)$ ) to have $\mathrm{B}$ (resp. A) at distance $m a$ of A (resp. B) and the three-sites probabilities $P_{\text {AAA }}(m, k)$, $P_{\mathrm{ABA}}(m, k), P_{\mathrm{BAA}}(m, k)$ and $P_{\mathrm{BBA}}(m, k)$, where $P_{\mathrm{ABA}}(m, k)$ is for example the probability 
to have $\mathrm{A}$ at site $n \mathbf{a}, \mathrm{B}$ at site $(m+n) \mathbf{a}$ and $\mathrm{A}$ at site $(k+n) \mathbf{a}$. With our notations

$$
\begin{aligned}
P_{\mathrm{AB}}(m) & =\left\langle\sigma_{n}^{\mathrm{A}} \sigma_{n+m}^{\mathrm{B}}\right\rangle_{n} \\
P_{\mathrm{ABA}}(m, k) & =\left\langle\sigma_{n}^{\mathrm{A}} \sigma_{n+m}^{\mathrm{B}} \sigma_{n+k}^{\mathrm{A}}\right\rangle_{n} \quad \text { etc. }
\end{aligned}
$$

Some of the properties of these pair and triplet coefficients used here are recalled in appendix $B[7,8]$ for a centrosymmetric structure.

By using these definitions, (24) becomes

$$
F=\left\langle\sigma_{n}^{\mathrm{A}}\right\rangle_{n} F_{\mathrm{A}}+\left\langle\sigma_{n}^{\mathrm{B}}\right\rangle_{n} F_{\mathrm{B}}-2 i \pi \mathbf{s} \cdot\left\langle\sum_{k}\left(\sigma_{n}^{\mathrm{A}} \sigma_{k}^{\mathrm{A}} \mathbf{u}_{n}^{k}\right) F_{\mathrm{A}}+\left(\sigma_{n}^{\mathrm{A}} \sigma_{k}^{\mathrm{B}} \mathbf{u}_{n}^{k}\right) F_{\mathrm{B}}\right\rangle_{n}
$$

or, after a $k \rightarrow k+n$ substitution, with the equality $\mathbf{u}_{n}^{n+k}=\mathbf{u}_{0}^{k}$

$$
F=x_{\mathrm{A}} F_{\mathrm{A}}+x_{\mathrm{B}} F_{\mathrm{B}}-2 i \pi \mathbf{s} \cdot \sum_{k}\left(P_{\mathrm{AA}}(k) F_{\mathrm{A}}+P_{\mathrm{BA}}(k) F_{\mathrm{B}}\right) \mathbf{u}_{0}^{k},
$$

which yields

$$
F=x_{\mathrm{A}} F_{\mathrm{A}}+x_{\mathrm{B}} F_{\mathrm{B}}-2 i \pi \mathbf{s} \cdot\left(F_{\mathrm{A}}-F_{\mathrm{B}}\right) \sum_{k} P_{\mathrm{BA}}(k) \mathbf{u}_{0}^{k}
$$

using (17) and the relationship $P_{\mathrm{AA}}(k)+P_{\mathrm{BA}}(k)=x_{\mathrm{A}}$.

Up to first order in displacement, the intensities of the Bragg spots are thus given by

$$
|F|^{2}=\left(x_{\mathrm{A}} F_{\mathrm{A}}+x_{\mathrm{B}} F_{\mathrm{B}}\right)^{2}
$$

with our hypothesis that the molecules are centrosymmetric.

The first term of (25) substituted into (23) gives the diffuse scattering due to short range order (S.R.O.) which is expressed as (see for example Warren [5])

$$
Y_{\text {S.R.o. }}(\mathrm{s})=x_{\mathrm{A}} x_{\mathrm{B}}\left(F_{\mathrm{A}}-F_{\mathrm{B}}\right)^{2} \sum_{m} \alpha(m) \exp (2 i \pi \mathrm{s} . m \mathbf{a})
$$

in which the coefficients $\alpha(m) \equiv\left(1-\frac{P_{\mathrm{BA}}(m)}{x_{\mathrm{A}} x_{\mathrm{B}}}\right)$ are the so-called Warren-Cowley S.R.O. parameters. If the system is completely disordered, $\alpha(0)=1$ and $\alpha(m)=0$ for $m \neq 0$, (30) gives the so-called Laue scattering.

$$
Y_{\text {S.R.o. }}(\mathrm{s})=x_{\mathrm{A}} x_{\mathrm{B}}\left(F_{\mathrm{A}}-F_{\mathrm{B}}\right)^{2} .
$$

By using (18) and (19), the second term of (25) is rewritten,

$$
\begin{aligned}
& 2 i \pi \mathrm{s} \cdot\left\{\left\langle\sum_{k} \sigma_{n}^{\mathrm{A}} \sigma_{n+m}^{\mathrm{A}} \sigma_{k}^{\mathrm{A}} \mathbf{u}_{n}^{k}\right\rangle_{n} F_{\mathrm{A}}\left(F^{*}-F_{\mathrm{A}}\right)-\left\langle\sum_{k} \sigma_{n+m}^{\mathrm{A}} \sigma_{n}^{\mathrm{A}} \sigma_{k}^{\mathrm{A}} \mathbf{u}_{n+m}^{k}\right\rangle_{n} F_{\mathrm{A}}\left(F-F_{\mathrm{A}}\right)\right. \\
& +\left\langle\sum_{k} \sigma_{n}^{\mathrm{A}} \sigma_{n+m}^{\mathrm{B}} \sigma_{k}^{\mathrm{A}} \mathbf{u}_{n}^{k}\right\rangle_{n} F_{\mathrm{A}}\left(F^{*}-F_{\mathrm{B}}\right)-\left\langle\sum_{k} \sigma_{n+m}^{\mathrm{A}} \sigma_{n}^{\mathrm{B}} \sigma_{k}^{\mathrm{A}} \mathbf{u}_{n+m}^{k}\right\rangle_{n} F_{\mathrm{A}}\left(F-F_{\mathrm{B}}\right) \\
& +\left\langle\sum_{k} \sigma_{n}^{\mathrm{B}} \sigma_{n+m}^{\mathrm{A}} \sigma_{k}^{\mathrm{A}} \mathbf{u}_{n}^{k}\right\rangle_{n} F_{\mathrm{B}}\left(F^{*}-F_{\mathrm{A}}\right)-\left\langle\sum_{k} \sigma_{n+m}^{\mathrm{B}} \sigma_{n}^{\mathrm{A}} \sigma_{k}^{\mathrm{A}} \mathbf{u}_{n+m}^{k}\right\rangle_{n} F_{\mathrm{B}}\left(F-F_{\mathrm{A}}\right) \\
& \left.+\left\langle\sum_{k} \sigma_{n}^{\mathrm{B}} \sigma_{n+m}^{\mathrm{B}} \sigma_{k}^{\mathrm{A}} \mathbf{u}_{n}^{k}\right\rangle_{n} F_{\mathrm{B}}\left(F^{*}-F_{\mathrm{B}}\right)-\left\langle\sum_{k} \sigma_{n+m}^{\mathrm{B}} \sigma_{n}^{\mathrm{B}} \sigma_{k}^{\mathrm{A}} \mathbf{u}_{n+m}^{k}\right\rangle_{n} F_{\mathrm{B}}\left(F-F_{\mathrm{B}}\right)\right\}
\end{aligned}
$$


By the use of the relationships (26) and a $k \rightarrow k+n$ substitution, (32) becomes

$$
\begin{aligned}
2 i \pi \mathrm{s} \cdot\left\{\sum_{k}\{\right. & \left.P_{\mathrm{AAA}}(m, k) F_{\mathrm{A}}\left(F^{*}-F_{\mathrm{A}}\right) \mathbf{u}_{0}^{k}-P_{\mathrm{AAA}}(m, k) F_{\mathrm{A}}\left(F-F_{\mathrm{A}}\right) \mathbf{u}_{m}^{k}\right\} \\
& +\sum_{k}\left\{P_{\mathrm{ABA}}(m, k) F_{\mathrm{A}}\left(F^{*}-F_{\mathrm{B}}\right) \mathbf{u}_{0}^{k}-P_{\mathrm{BAA}}(m, k) F_{\mathrm{A}}\left(F-F_{\mathrm{B}}\right) \mathbf{u}_{m}^{k}\right\} \\
& +\sum_{k}\left\{P_{\mathrm{BAA}}(m, k) F_{\mathrm{B}}\left(F^{*}-F_{\mathrm{A}}\right) \mathbf{u}_{0}^{k}-P_{\mathrm{ABA}}(m, k) F_{\mathrm{B}}^{*}\left(F-F_{\mathrm{A}}\right) \mathbf{u}_{m}^{k}\right\} \\
& \left.+\sum_{k}\left\{P_{\mathrm{BBA}}(m, k) F_{\mathrm{B}}\left(F^{*}-F_{\mathrm{B}}\right) \mathbf{u}_{0}^{k}-P_{\mathrm{BBA}}(m, k) F_{\mathrm{B}}\left(F-F_{\mathrm{B}}\right) \mathbf{u}_{m}^{k}\right\}\right\} .
\end{aligned}
$$

By considering the four symmetry relationships of the type $P_{\mathrm{ABA}}(m, k)=$ $P_{\mathrm{BAA}}(m, m-k)$, (33) becomes

$$
2 i \pi \mathbf{s} \cdot \sum_{k}\left\{C(m, k) \mathbf{u}_{-m}^{-k}-C(m, k)^{*} \mathbf{u}_{m}^{k}\right\}
$$

with

$$
\begin{aligned}
C(m, k)= & \left(F^{*}-F_{\mathrm{A}}\right)\left(P_{\mathrm{AAA}}(m, k) F_{\mathrm{A}}+P_{\mathrm{ABA}}(m, k) F_{\mathrm{B}}\right) \\
& +\left(F^{*}-F_{\mathrm{B}}\right)\left(P_{\mathrm{BAA}}(m, k) F_{\mathrm{A}}+P_{\mathrm{BBA}}(m, k) F_{\mathrm{B}}\right) .
\end{aligned}
$$

If we neglect the $u$ linear dependence of $F^{*}$, which gives second order terms in displacement in (34), $C(m, k)$ becomes

$$
\begin{aligned}
C(m, k)=\left(F_{\mathrm{A}}-F_{\mathrm{B}}\right)\left\{x_{\mathrm{A}}\left(P_{\mathrm{BAA}}(m, k) F_{\mathrm{A}}+P_{\mathrm{BBA}}(m, k) F_{\mathrm{B}}\right)-\right. \\
\left.-x_{\mathrm{B}}\left(P_{\mathrm{AAA}}(m, k) F_{\mathrm{A}}+P_{\mathrm{ABA}}(m, k) F_{\mathrm{B}}\right)\right\},
\end{aligned}
$$

which, by combining with the (B3), (B4) and (B7) relationships of appendix B, may be expressed as

$$
\begin{aligned}
C(m, k)=F_{\mathrm{B}}\left(F_{\mathrm{A}}-F_{\mathrm{B}}\right)\left(P_{\mathrm{BA}}(k)-x_{\mathrm{A}} x_{\mathrm{B}}\right)+\left(F_{\mathrm{A}}-F_{\mathrm{B}}\right)^{2} P_{\mathrm{ABA}}(m, m-k)- \\
-x_{\mathrm{B}}\left(F_{\mathrm{A}}-F_{\mathrm{B}}\right)^{2} P_{\mathrm{AA}}(m-k) .
\end{aligned}
$$

Considering that

$$
\begin{aligned}
\mathbf{u}_{-m}^{-k}-\mathbf{u}_{m}^{k} & =\sum_{\mathbf{q}^{\prime}} \mathbf{u}_{\mathbf{q}^{\prime}}\left(\sin \left(2 \pi \mathbf{q}^{\prime}(k-m) \mathbf{a}+\varphi\right)-\sin \left(2 \pi \mathbf{q}^{\prime}(m-k) \mathbf{a}+\varphi\right)\right) \\
& =2 \cos \varphi \sum_{\mathbf{q}^{\prime}} \mathbf{u}_{\mathbf{q}^{\prime}} \sin \left(2 \pi \mathbf{q}^{\prime}(k-m) \mathbf{a}\right)
\end{aligned}
$$

the expression (34) then becomes

$$
4 i \pi \cos \varphi \mathbf{s} \cdot \sum_{k}\left\{C(m, k) \sum_{\mathbf{q}^{\prime}} \mathbf{u}_{\mathbf{q}^{\prime}} \sin \left(2 \pi \mathbf{q}^{\prime}(k-m)\right)\right\} .
$$

Thus, by substituting the second term of (25) in (23), one obtains

$$
Y_{\mathrm{W} . \mathrm{L} .}(\mathbf{s})=2 i \pi \mathbf{s} \cdot \sum_{m}\left\langle F_{n}\left(F^{*}-F_{n+m}^{*}\right) \mathbf{u}_{n}-F_{n+m}^{*}\left(F-F_{n}\right) \mathbf{u}_{n+m}\right\rangle_{n} \exp (2 i \pi \mathbf{s} m \mathbf{a})
$$


which may be expressed as

$$
\begin{aligned}
& Y_{\mathrm{W} . \mathrm{L} .}(\mathbf{s})=-4 i x_{\mathrm{A}} x_{\mathrm{B}} F_{\mathrm{B}}\left(F_{\mathrm{A}}-F_{\mathrm{B}}\right) \pi \cos \varphi \times \\
& \quad \times \mathbf{s} \cdot \sum_{\mathbf{q}^{\prime}} \mathbf{u}_{\mathbf{q}^{\prime}} \sum_{m, k} \alpha(k) \sin \left(2 \pi \mathbf{q}^{\prime}(k-m) \mathbf{a}\right) \exp (2 i \pi \mathbf{s} m \mathbf{a}) \\
& +4 i\left(F_{\mathrm{A}}-F_{\mathrm{B}}\right)^{2} \pi \cos \varphi \mathbf{s} \cdot \sum_{\mathbf{q}^{\prime}} \mathbf{u}_{\mathbf{q}^{\prime}} \sum_{m, k} P_{\mathrm{ABA}}(m, m-k) \sin \left(2 \pi \mathbf{q}^{\prime}(k-m) \mathbf{a}\right) \exp (2 i \pi \mathbf{s} m \mathbf{a}) \\
& -4 i x_{\mathrm{B}}\left(F_{\mathrm{A}}-F_{\mathrm{B}}\right)^{2} \pi \cos \varphi \mathbf{s} \cdot \sum_{\mathbf{q}^{\prime}} \mathbf{u}_{\mathbf{q}^{\prime}} \sum_{m, k} P_{\mathrm{AA}}(m-k) \sin \left(2 \pi \mathbf{q}^{\prime}(k-m) \mathbf{a}\right) \exp (2 i \pi \mathbf{s} m \mathbf{a}) .
\end{aligned}
$$

Using the parity properties of the pair and triplet coefficients, the third term of (40) vanishes and one gets

$$
\begin{aligned}
& Y_{\mathrm{W} . \mathrm{L} .}(\mathbf{s})=2 x_{\mathrm{A}} x_{\mathrm{B}} F_{\mathrm{B}}\left(F_{\mathrm{A}}-F_{\mathrm{B}}\right) \pi \cos \varphi \mathrm{s} \cdot \sum_{\mathbf{q}^{\prime}} \mathbf{u}_{\mathbf{q}^{\prime}} \sum_{k} \alpha(k) \exp \left(2 i \pi \mathbf{q}^{\prime} k \mathbf{a}\right) \times \\
& \quad \times \sum_{m}\left\{\exp \left(2 i \pi\left(\mathbf{s}+\mathbf{q}^{\prime}\right) m \mathbf{a}\right)-\exp \left(2 i \pi\left(\mathbf{s}-\mathbf{q}^{\prime}\right) m \mathbf{a}\right)\right\} \\
& +4\left(F_{\mathrm{A}}-F_{\mathrm{B}}\right)^{2} \pi \cos \varphi \mathbf{s} \cdot \sum_{\mathbf{q}^{\prime}} \mathbf{u}_{\mathbf{q}^{\prime}} \sum_{m, k} P_{\mathrm{ABA}}(m, k) \sin \left(2 \pi \mathbf{q}^{\prime} k \mathbf{a}\right) \sin (2 \pi \mathbf{s} m \mathbf{a}) .
\end{aligned}
$$

We can check that the $s$ dependence of (41) is antisymmetric about each reciprocal lattice node. The first term (which is non zero only when $\mathbf{s}=\mathbf{q}^{\prime} \pm \mathbf{Q}$, where $\mathbf{Q}$ is a reciprocal lattice vector) is analogous to that obtained in section 2 and originates from the correlation between the displacement of a molecule and the position of one impurity $\mathrm{A}$. This is the reason why it depends only on pair coefficients. The second term, which contains triplet coefficients, is due to the correlation between the displacement of a molecule and the position of two impurities A.

In order to evaluate the respective contributions of these two terms to the diffuse scattering, let us calculate $Y_{\text {W.L. }}$ (s) in a case of a complete disorder (i.e. for an uncorrelated system). The pair and triplet coefficients are respectively equal to

$$
P_{\mathrm{AB}}(0)=0 \text { and } P_{\mathrm{AB}}(m)=x_{\mathrm{A}} x_{\mathrm{B}} \text { for } m \neq 0
$$

and to $P_{\mathrm{ABA}}(m, 0)=x_{\mathrm{A}} x_{\mathrm{B}}, P_{\mathrm{ABA}}(0, k)=P_{\mathrm{ABA}}(m, m)=0$ and $P_{\mathrm{ABA}}(m, k)=x_{\mathrm{A}}^{2} x_{\mathrm{B}}$ for $m \neq k \neq 0$.

This gives

$$
\begin{aligned}
& Y_{\mathrm{W} . \mathrm{L} .}(\mathbf{s})=2 x_{\mathrm{A}} x_{\mathrm{B}} F_{\mathrm{B}}\left(F_{\mathrm{A}}-F_{\mathrm{B}}\right) \pi \cos \varphi \times \\
& \quad \times \mathbf{s} \cdot \sum_{\mathbf{q}^{\prime}} \mathbf{u}_{\mathbf{q}^{\prime}} \sum_{m}\left\{\exp \left(2 i \pi\left(\mathbf{s}+\mathbf{q}^{\prime}\right) m \mathbf{a}\right)-\exp \left(2 i \pi\left(\mathbf{s}-\mathbf{q}^{\prime}\right) m \mathbf{a}\right)\right\} \\
& \quad+2 x_{\mathrm{A}}^{2} x_{\mathrm{B}}\left(F_{\mathrm{A}}-F_{\mathrm{B}}\right)^{2} \pi \cos \varphi \mathbf{s} \cdot \sum_{\mathbf{q}^{\prime}} \mathbf{u}_{\mathbf{q}^{\prime}} \sum_{m}\left\{\exp \left(2 i \pi\left(\mathbf{s}+\mathbf{q}^{\prime}\right) m \mathbf{a}\right)-\exp \left(2 i \pi\left(\mathbf{s}-\mathbf{q}^{\prime}\right) m \mathbf{a}\right)\right\}
\end{aligned}
$$

where we can see that if $x_{\mathrm{A}}$ is small the second term is negligible. In a general situation, as emphasized by Cowley [6], the magnitude of a term which contains triplet coefficients is difficult to estimate. However in the case of the size-effect, which also involves such high order terms, Welberry shows by optical transform methods [9] that these terms have a small influence on the diffraction pattern. On physical grounds, in the framework of our model, it 
seems unlikely that the second term of (41) can significantly contribute to the diffuse scattering if the average distances between impurities $\mathrm{A}, a / x_{\mathrm{A}}$, is larger than the correlation lengh $\xi$ of the displacement wave induced by an impurity. When the second term of (41) is neglected, the diffuse scattering is thus given by

with

$$
Y_{\text {Diff }}(\mathbf{s})=Y_{\text {S.R.O. }}(\mathbf{s})+Y_{\text {W.L. }}(\mathbf{s}) \text {, }
$$

$$
Y_{\text {S.R.o. }}(\mathbf{s})=x_{\mathrm{A}} x_{\mathrm{B}}\left(F_{\mathrm{A}}-F_{\mathrm{B}}\right)^{2} \sum_{m} \alpha(m) \exp (2 i \pi \mathbf{s} \cdot m \mathbf{a})
$$

and

$$
\begin{aligned}
Y_{\mathrm{W} . \mathrm{L} .}(\mathbf{s})=2 x_{\mathrm{A}} x_{\mathrm{B}} F_{\mathrm{B}}\left(F_{\mathrm{A}}-F_{\mathrm{B}}\right) \pi \cos \varphi \mathbf{s} \cdot \sum_{\mathbf{q}^{\prime}} \mathbf{u}_{\mathbf{q}^{\prime}} \sum_{k} \alpha(k) \exp \left(2 i \pi \mathbf{q}^{\prime} k \mathbf{a}\right) \times \\
\quad \times \sum_{m}\left\{\exp \left(2 i \pi\left(\mathbf{s}+\mathbf{q}^{\prime}\right) m \mathbf{a}\right)-\exp \left(2 i \pi\left(\mathbf{s}-\mathbf{q}^{\prime}\right) m \mathbf{a}\right)\right\}
\end{aligned}
$$

$Y_{\text {W.L. }}$ (s) has a non-zero value only if $\mathbf{s} \pm \mathbf{q}^{\prime}=\mathbf{Q}$, which becomes finally

$$
\begin{aligned}
& Y_{\text {W.L. }}\left(\mathbf{Q}+\mathbf{q}^{\prime}\right)=-2 x_{\mathrm{A}} x_{\mathrm{B}} F_{\mathrm{B}}\left(F_{\mathrm{A}}-F_{\mathrm{B}}\right) \pi \cos \varphi \mathbf{s} \cdot \mathbf{u}_{\mathbf{q}^{\prime}} \sum_{k} \alpha(k) \exp \left(2 i \pi \mathbf{q}^{\prime} k \mathbf{a}\right) \\
& Y_{\text {W.L. }}\left(\mathbf{Q}+\mathbf{q}^{\prime}\right)=+2 x_{\mathrm{A}} x_{\mathrm{B}} F_{\mathrm{B}}\left(F_{\mathrm{A}}-F_{\mathrm{B}}\right) \pi \cos \varphi \mathbf{s} \cdot \mathbf{u}_{\mathbf{q}^{\prime}} \sum_{k} \alpha(k) \exp \left(2 i \pi \mathbf{q}^{\prime} k \mathbf{a}\right) .
\end{aligned}
$$

\section{Discussion.}

4.1 ReleVANT PaRAMETERS OF THE WhITE LINE EFFECT. - First, let us recall that the previous results are obtained for an alloyed chain i.e. for a one-dimensional model. The generalization of the calculations to a three dimensional lattice is straightforward if we suppose that the disorder and the distortion are uncorrelated from chain to chain. Each chain scatters independently and the diffuse scattering intensity simply adds. The expressions (42) and (43) remain unchanged.

Let us now discuss the significance of these formulae. The diffuse scattering intensity calculated consists of two terms. The first one $Y_{\text {S.R.o. }}$ (s) describes a monotonic scattering with smooth variations in reciprocal space depending on the degree of order of the system. This scattering will appear as a modulated «background » on $\mathrm{X}$-ray patterns. Due to the pinning of displacement waves to the impurities, new effects occur via the second term $Y_{\text {W.L. }}$ (s). If we assume that the wave vector distribution of the $\mathbf{u}_{\mathbf{q}^{\prime}}$ has a small width ( $2 \pi q \xi \gg 1$ condition), this effect will be observed only in the vicinity of the $\mathbf{Q}-\mathbf{q}$ and $\mathbf{Q}+\mathbf{q}$ reciprocal positions in the form of diffuse streaks. If the quantity $F_{\mathrm{B}}\left(F_{\mathrm{A}}-F_{\mathrm{B}}\right) \cos \varphi \mathrm{s} . \mathbf{u}_{\mathrm{q}}$, is positive (negative) the white lines will appear for wave vectors larger (smaller) than those of the Bragg reflections. As an exception, if $\mathbf{q}=\mathbf{a}^{*} / 2$, the black and white diffuse lines overlap and $Y_{\text {W.L. }}$ (s) no longer contributes. Diffuse lines of weaker intensity, due to the second order terms in displacement not considered here, will be observed.

Let us remark that $Y_{\mathrm{W} . \mathrm{L} .}$ (s) contains the $x_{\mathrm{A}} x_{\mathrm{B}}\left(F_{\mathrm{A}}-F_{\mathrm{B}}\right) \sum_{k} \alpha(k) \exp \left(2 i \pi \mathbf{q}^{\prime} k \mathbf{a}\right)$ factor, which also enters the expression of the short range order diffuse scattering. This expected result shows that the white line effect occurs only for scattering vectors where the Laue «background » is non zero.

The most original feature of this effect, due to the linearity with respect to the amplitude of the displacement, is the relevance of the signs of the $F_{\mathrm{B}}\left(F_{\mathrm{A}}-F_{\mathrm{B}}\right), \cos \varphi$ and $\mathbf{s} . \mathbf{u}_{\mathrm{q}}$ factors on the total scattered intensity. Let us examine their effect successively. 

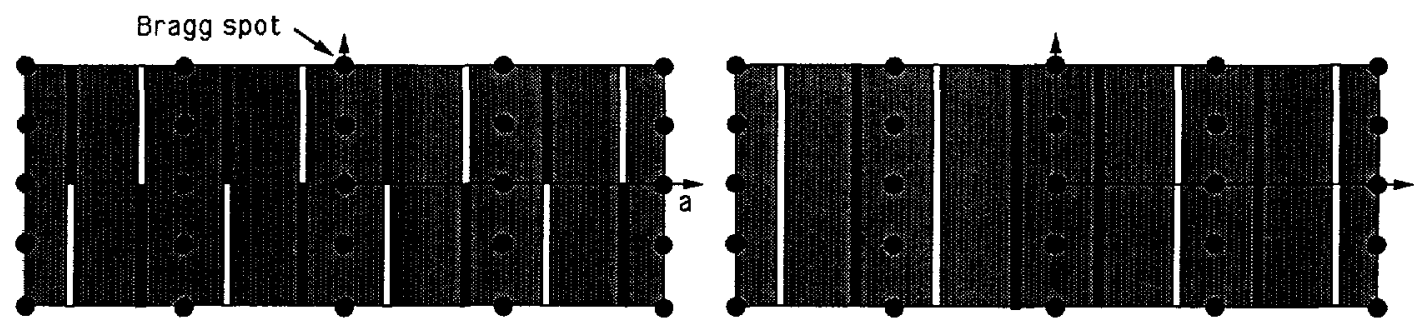

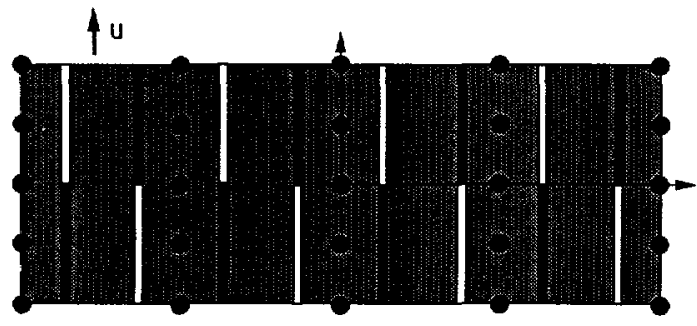

u transverse

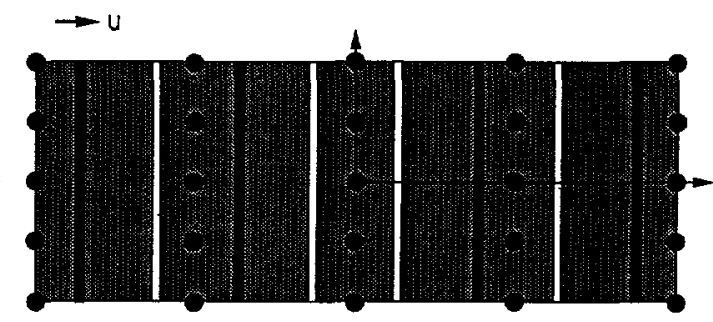

u longltudinal

Fig. 6. - Locations of white and black diffuse lines for a transverse modulation (left) and a longitudinal modulations (right). The grey background represents a constant Laue scattering. The quantity $F_{\mathrm{B}}\left(F_{\mathrm{A}}-F_{\mathrm{B}}\right) \cos \varphi$ is negative in the upper figures and positive in the lower ones.

- The effect of the $\mathbf{s} . \mathbf{u}_{\mathbf{q}}$ term can be understood by studying two simple cases sketched in figure 6, when a constant Laue scattering is assumed. If the distortion wave has a longitudinal polarization (i.e. if $\mathbf{u}_{\mathbf{q}^{\prime}}$ is parallel to $\mathbf{q}$ ), each diffuse line is continuous and does not present any inversion of intensity. On the other hand if the distortion is transverse ( $\mathbf{u}_{\mathbf{q}^{\prime}}$ perpendicular to $\mathbf{q}$ ) the $\mathbf{s} . \mathbf{u}_{\mathbf{q}^{\prime}}$ term changes sign when the scattering vector $\mathbf{s}$ is perpendicular to $\mathbf{u}_{\mathbf{q}^{\prime}}$. At these inversions, the diffuse line intensity vanishes. This feature provides a way to measure the polarization of the displacement wave by looking at the locus of the inversions of intensity. To study the influence of the other terms we shall consider the case of a longitudinal polarization.

- The quantity $F_{\mathrm{B}}\left(F_{\mathrm{A}}-F_{\mathrm{B}}\right)$ only depends on the nature of the scattering species. If atoms were considered, this factor will change sign according to the weight of the impurity $A$ with respect to the atoms $B$. Everything being equal, a lattice in which the $A$ atoms are the impurities gives a diffuse scattering pattern with white lines positions inverted with respect to the pattern obtained from the same lattice where the $B$ atoms are the impurities. If the scattering species are complex molecules, we have to compute carefully the $F_{\mathrm{B}}\left(F_{\mathrm{A}}-F_{\mathrm{B}}\right)$ quantity, which can change sign when $s$ varies. We shall come back to its effect in the next section.

- The influence of the phase term $\cos \varphi$ has already been discussed in section 2.1. If the phase of the displacement wave has the special values $\pm \pi / 2$, the white line effect disappears and second order terms become dominant, as in a pure compound. If the phase differs from these two values, the white line's position depends on the sign of $\cos \varphi$. Note that as expected from a kinematic treatment of the diffraction, we do not obtain information on the absolute phase of the modulation wave, but on the relative phase between the distortion and the composition waves. Indeed, as emphasized before, this feature is also present in the sizeeffect and permits one to obtain local information of the deformation field around an impurity.

4.2 EXPERIMENTAL EVIDENCE OF THE WHITE LINE EFFECT. - Now we can come back to the $X$-ray patterns presented figure 1 in the introduction. HMTSF-TCNQ, whose constituting 
molecules are displayed figure 7 , crystallizes in the $C_{2 / m}$ monoclinic space group with $a=21.99 \AA, b=12.573 \AA, C=3.89 \AA$ and $\beta=90.29^{\circ}[10]$, $c$ being the chain axis. This compound exhibits a large regime of $1 D$ fluctuations at $2 \mathbf{k}_{\mathrm{f}}=0.37 \mathbf{c}^{*}$ visible in the form of diffuse lines (Fig. 1a), which condense into very weak satellites reflections at the reduced wave vector $(0,0.37,0)$ in the $10 \mathrm{~K}-25 \mathrm{~K}$ temperature range [1]. The direction of polarization $\mathbf{e}$ of the lattice distortion in the regime of $1 \mathrm{D}$ fluctuations has already been obtained by Yamaji et al. [11] from a fit of the intensity of the $\pm 2 \mathbf{k}_{\mathrm{f}}$ diffuse lines, measured on X-ray photographs similar to those of figure 1. This direction was found to be $\mathbf{e}=0.19(8) \mathbf{a}+$ $0.06(15) \mathbf{b}+\mathbf{c}$.

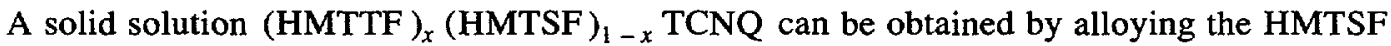
molecule with its sulfur analogue HMTTF also shown figure 7 . Figure $1 \mathrm{~b}$ presents the X-ray pattern obtained at $25 \mathrm{~K}$ from an alloy with a nominal concentration of $x=0.25$ (in fact $x=0.05$ from microprobe analysis). It exhibits two essential features. First, a modulated diffuse background is visible, with maxima located on three broad diffuse stripes (Fig. 1b) running along the $\mathrm{c}^{*}$ direction and secondary weak maxima between the $\mathcal{l}=$ constant layers of Bragg spots. At the $(\ell+0.37) \mathrm{c}^{*}$ scattering vectors, white lines appear at the expense of this background, without any inversion of intensity along the $\mathbf{b}^{*}$ direction. The normal black diffuse lines are observed at the $(\ell-0.37) c^{*}$ scattering vectors. These lines are thin with half-width at half-maximum (H.W.H.M.) of $0.007 \AA^{-1}$.

In this case, the short range order diffuse scattering is proportional to $x(1-x)$ $\left(F_{\text {HMTTF }}-F_{\text {HMTSF }}\right)^{2}$. The quantity $\left(F_{\text {HMTTF }}-F_{\text {HMTSF }}\right)$ has complex variations in reciprocal space which are mainly due to the Se/S atomic substitution on the donor molecule and which, to a first approximation, corresponds to the Fourier transform of a rectangle formed by « atoms » of scattering factors $\left(f_{s}-f_{S e}\right)$. Computer simulations are then needed to evaluate the $s$ variations of the Laue scattering. They show that, for the orientation of our crystal, the «monotonic » Laue scattering forms large stripes along the $\mathbf{c}^{*}$ direction, as observed on the $\mathrm{X}$-ray pattern of figure $1 \mathrm{~b}$. The slight increase of the diffuse scattering midway between the $\ell=$ constant Bragg layers is the signature of a weak short range order in the direction, probably due to repulsion between impurities.

In the framework of our model, we propose that around each HMTTF impurity, a periodic lattice distortion is pinned, with the $q=2 \mathbf{k}_{\mathrm{f}}=0.37 \mathbf{c}^{*}$ wave vector and a correlation length of $\xi=60 \AA$ (obtained by assuming a Gaussian experimental resolution and a Lorentzian shape for the $\mathbf{u}_{\mathbf{q}^{\prime}}$ correlation function).

On the X-ray pattern of figure $1 \mathrm{~b}$, which shows a part of the reciprocal space with small $h$, the white lines do not present any inversion of intensity. Therefore the polarization of the
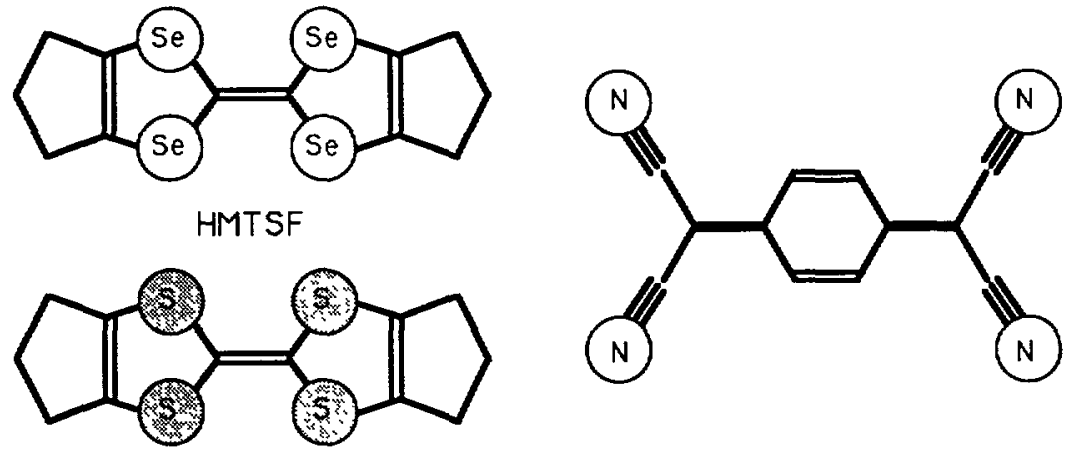

HMTTF

TCNQ

Fig. 7. - Molecular structure of HMTSF, HMTTF, TCNQ. 
distortion is mainly directed along the c direction, which is consistent with the results of reference [11].

The sign of the $F_{\mathrm{HMTSF}}\left(F_{\mathrm{HMTTF}}-F_{\mathrm{HMTSF}}\right)$ quantity is that of the $f_{\mathrm{Se}}\left(f_{\mathrm{S}}-f_{\mathrm{Se}}\right)$ one, if the slight differences of intramolecular parameters can be neglected (which is justified at low scattering angles). This quantity has the same $s$-variation as the « monotonic » Laue scattering and its sign is negative.

We can now determine the sign of the third parameter, $\cos \varphi$. As $\mathbf{s} . \mathbf{u}_{\mathbf{q}^{\prime}}$ is positive, $F_{\text {HMTSF }}\left(F_{\text {HMTTF }}-F_{\text {HMTSF}}\right)$ negative and the white lines appear at $+2 \mathbf{k}_{\mathrm{f}}$ for $\ell>0$, we can deduce from (44) that $\cos \varphi$ is negative. In that case, figure 8 gives a direct space representation of a modulation pinned on a HMTTF molecular impurity for $\varphi=\pi$ (for this phase the impurity is not displaced).

Now, let us discuss the validity of the approximation made in this calculations. The first one concerns the hypothesis of small displacements : $2 \pi s u \ll 1$. The magnitude of the displacements of the donor molecule involved in the Peierls instability is not available for HMTSFTCNQ but it has been determined for the parent compound TTF-TCNQ [12]. The order of magnitude of the displacements of the atoms of the two species is $u \approx 0.01 \AA(0.008 \AA$ for TTF, $0.02 \AA$ for the quinoid ring of TCNQ and $0.01 \AA$ for its cyanomethylene groups). The reciprocal space region accessible for $\mathrm{X}$-rays of a wavelength of $1.542 \AA$, corresponds to scattering wave vectors smaller than $0.6 \AA^{-1}$. This gives $2 \pi Q u<0.04$, which justifies our approximation that the second order terms in displacement can be neglected. The second hypothesis, which is not essential to account for the white line effect, is that for the alloy studied $x \cdot \xi / c=0.75<1$. This means that the term containing triplet probabilities in (41) is not important.

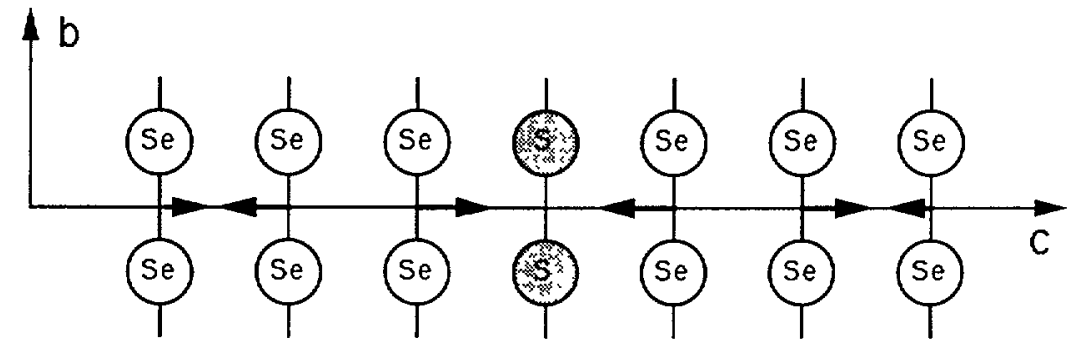

Fig. 8. - Representation of the modulation of the positions of the HMTSF molecules around a HMTTF impurity. The directions of displacement sketched correspond to the experimental situation of (HMTTF $)_{0.05}$ (HMTSF $)_{095}$ TCNQ.

4.3 Physical INTERPRETATION. - The physical reasons for the appearance of such pinning effects in these $1 \mathrm{D}$ compounds can be briefly discussed. The introduction of an impurity in a $1 \mathrm{D}$ electron gas is equivalent to add locally a perturbating potential in the lattice. The 1D electron gas will screen this potential with a response proportional to the dielectric constant of the gas. In a $1 \mathrm{D}$ metallic state it is well known that this response shows a maximum at the $2 \mathbf{k}_{\mathrm{f}}$ wave vector. A charge density wave will be induced around the impurity with the $2 \mathbf{k}_{\mathrm{f}}$ wave vector. This Friedel oscillation, coupled to the lattice, will give rise to a periodic lattice distortion, also pinned on the impurity with the same wave vector [13]. As this distortion is $90^{\circ}$ out-of-phase with the charge density wave, its relative phase $\varphi$ with respect to the impurity will be 0 or $\pi$, depending on the type of defect. From the observation of $2 \mathbf{k}_{\mathrm{f}}$ white lines in (HMTTF $)_{0.05}$ (HMTSF $)_{0.95}$ TCNQ, our data present a direct evidence of $2 \mathbf{k}_{\mathrm{f}}$ lattice distortions with $\varphi=\pi$ coupled to charge density wave oscillations induced by molecular impurities on the donor stacks. A more complete interpretation emphasizing the size effect due to the impurity will be published elsewhere [14]. 


\section{Conclusion.}

In this paper, we have presented a simple model to explain the appearance of white diffuse lines in the X-ray pattern of solid solutions of charge transfer one-dimensional salts. This effect is shown to result from a coherence between a periodic lattice distortion and the impurity position. Due to the linearity of the effect, new information can be extracted from the location of these white lines such as : the polarisation of the lattice distortion and the phase of the displacement wave with respect to the impurity. In addition, in 1D systems where several types of stacks can exhibit a charge density wave instability, this effect simply permits the assignment of the instability to the stack where molecular substitutions produce the white line effect. In this respect, let us mention that this effect has also been observed on the $4 \mathbf{k}_{\mathrm{f}}$ diffuse scattering of the TTF rich side of TTF(TSF)-TCNQ alloys [1,3] and on both $2 \mathbf{k}_{\mathrm{f}}$ and $4 \mathbf{k}_{\mathrm{f}}$ scattering of the HMTTF rich side of HMTTF(HMTSF)-TCNQ alloys [14]. As for the $2 k_{\mathrm{f}}$ diffuse scattering of HMTSF(HMTTF)-TCNQ alloys, it allows to assign unambiguously these $2 \mathbf{k}_{\mathrm{f}}$ and(or) $4 \mathbf{k}_{\mathrm{f}}$ instabilities to the donor stacks.

Finally it is worth noting that such negative interferences are generally expected when lattice distortions occur in quadrature with molecular disorder. Somewhat similar features have been observed in liquid crystals [15] (for example in terephtal bis butyl aniline single crystals (TBBA)) and have been attributed to a linear defect consisting of a localized vacancy surrounded by a delocalized interstitial.

\section{Acknowledgements.}

We are indebted to S. Megtert, R. Moret, E. Dubois-Violette, B. Pansu and J. Friedel for fruitful discussions. $Q$. Liu wrote the computer simulation program of the Laue scattering used in section 4.2 and Y.-S. Huang performed the microprobe analysis of the (HMTTF $)_{x}$ (HMTSF $)_{1-\lambda}$ TCNQ crystal investigated here. This solid solution has been synthesized by $\mathrm{K}$. Bechgaard and kindly provided to us by B. Hamzic.

\section{Appendix A.}

The amplitude of the wave diffracted by a lattice of $N$ atoms with two correlated modulations is given by

$$
A(s)=\sum_{n} f(1+\eta \sin (2 \pi q n a+\psi)) \exp (-2 i \pi s(n a+u \sin (2 \pi q n a+\varphi))) .
$$

By using the Fourier development of $\exp (i z \sin \theta)$

$$
\exp (i z \sin \theta)=\sum_{n} J_{n^{\prime}}(z) \exp \left(i n^{\prime} \theta\right),
$$

where $J_{n^{\prime}}(z)$ is the Bessel function of $n^{\prime}$-th order, (A1) becomes

$\sum_{n} f(1+\eta \sin (2 \pi q n a+\psi))\left\{\sum_{n^{\prime}} J_{n^{\prime}}(-2 \pi s u) \exp \left(2 i \pi n^{\prime} q n a+i n^{\prime} \varphi\right)\right\} \exp (-2 i \pi s n a)$

By using $\sin x=(\exp (i x)-\exp (-i x)) / 2 i$, this sum can be divided in three terms

$$
\begin{gathered}
\sum_{n, n^{\prime}} f\left\{J_{n^{\prime}}(-2 \pi s u) \exp \left(i n^{\prime} \varphi\right)\right\} \exp \left(-2 i \pi\left(s-n^{\prime} q\right) n a\right) \\
+\frac{1}{2 i} \sum_{n, n^{\prime}} f\left\{J_{n^{\prime}}(-2 \pi s u) \eta \exp (i \psi) \exp \left(i n^{\prime} \varphi\right)\right\} \exp \left(-2 i \pi\left(s-\left(n^{\prime}+1\right) q\right) n a\right) \\
-\frac{1}{2 i} \sum_{n, n^{\prime}} f\left\{J_{n^{\prime}}(-2 \pi s u) \eta \exp (-i \psi) \exp \left(i n^{\prime} \varphi\right)\right\} \exp \left(-2 i \pi\left(s-\left(n^{\prime}-1\right) q\right) n a\right)
\end{gathered}
$$


The summations over $n$ are non-zero only if the terms in the exponential are multiple of $2 \pi$. i.e. if $s=Q+m q$, where $Q=h / a$ and $m$ is an integer. The coefficient $A_{m}(s)$ of the $s=Q+m q$ term is then given by

$$
\begin{aligned}
A_{m}(s)=f & \exp (i m(\varphi+\pi)) \times \\
& \times\left\{J_{m}(2 \pi s u)-\frac{\eta}{2 i}\left(J_{m-1}(2 \pi s u) \exp (-i \Phi)-J_{m+1}(2 \pi s u) \exp (i \Phi)\right)\right\}
\end{aligned}
$$

where we pose $\Phi=\varphi-\psi$ and where we use the relation $J_{m}(-x)=(-1)^{m} J_{m}(x)$.

If $q$ is incommensurate, in order to avoid some high order interference effects, the intensity of a peak at position $s=Q+m q$ is

$$
\begin{aligned}
I(s=Q & +m q)=N^{2} f^{2} \times \\
& \times\left|J_{m}(2 \pi s u)-\frac{\eta}{2 i}\left\{J_{m-1}(2 \pi s u) \exp (-i \Phi)-J_{m+1}(2 \pi s u) \exp (i \Phi)\right\}\right|^{2},
\end{aligned}
$$

which gives

$$
\begin{aligned}
I(s= & Q+m q)=N^{2} f^{2}\left[J_{m^{2}}(2 \pi s u)+\eta \sin \Phi\left\{J_{m}(2 \pi s u)\left(J_{m-1}(2 \pi s u)+J_{m+1}(2 \pi s u)\right)\right\}+\right. \\
& \left.+\frac{\eta^{2}}{4}\left\{J_{m-1^{2}}(2 \pi s u)+J_{m+1^{2}}(2 \pi s u)-2 J_{m-1}(2 \pi s u) J_{m+1}(2 \pi s u) \cos (2 \Phi)\right\}\right] \text { (A9) }
\end{aligned}
$$

In section 1.1 we use this formula with the approximation $J_{m}(2 \pi s u) \approx(\pi s u)^{m} / m$ ! when $2 \pi s u \ll 1$. It can be seen that for $|m|=1$, the $\eta$-term is dominant. It gives a $+q l-q$ asymmetry of intensity. For $|m|>1$ the last term of (A9) is preponderant. It does not give rise to such an asymmetry.

\section{Appendix B.}

The pair and triplet probabilities verify the following relationships

$$
\begin{gathered}
P_{\mathrm{AB}}(m)+P_{\mathrm{AA}}(m)=x_{\mathrm{A}} \\
P_{\mathrm{BA}}(m)+P_{\mathrm{BB}}(m)=x_{\mathrm{B}} \\
P_{\mathrm{AAA}}(m, k)+P_{\mathrm{ABA}}(m, k)=P_{\mathrm{AA}}(m) \text { and } P_{\mathrm{BBA}}(m, k)+P_{\mathrm{BAA}}(m, k)=P_{\mathrm{BA}}(m) \\
P_{\mathrm{AAA}}(m, k)+P_{\mathrm{BAA}}(m, k)=P_{\mathrm{AA}}(m-k) \text { and } P_{\mathrm{BBA}}(m, k)+P_{\mathrm{ABA}}(m, k)=P_{\mathrm{BA}}(m-k)
\end{gathered}
$$

Moreover, if we assume that the lattice is centrosymmetric, new relations are obeyed.

$$
P_{\mathrm{AB}}(m)=P_{\mathrm{AB}}(-m)=\mathrm{P}_{\mathrm{BA}}(m),
$$

and, with the help of figure 9

$$
\begin{array}{ll}
P_{\mathrm{AAA}}(m, k) & =P_{\mathrm{AAA}}(m, m-k) \\
P_{\mathrm{ABA}}(m, k) & =P_{\mathrm{BAA}}(m, m-k) \\
P_{\mathrm{BBA}}(m, k) & =P_{\mathrm{BBA}}(m, m-k) \\
P_{\mathrm{ABA}}(-m,-k) & =P_{\mathrm{ABA}}(m, k) .
\end{array}
$$




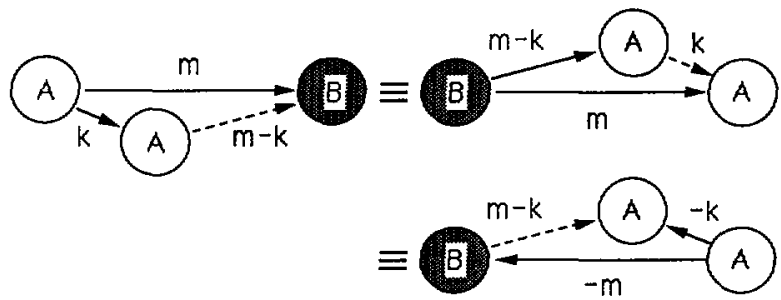

Fig. 9. - Equivalence of three centrosymmetric situations showing that $P_{\mathrm{ABA}}(m, k)=$ $P_{\mathrm{BAA}}(m, m-k)=P_{\mathrm{ABA}}(-m,-k)$.

\section{References}

[1] Pouget J. P., Structural Instabilities, chapter 3 in Highly Conducting Quasi One Dimensional Organic Crystals, Semiconductors and Semi metals, vol. 27, E. M. Conwell ed. (Academic Press, 1988) p. 87.

[2] CoMES R. and SHIRANe G., X-ray and Neutron Scattering from One-Dimensional Solids J. T.

Devreese, R. P. Evrard and V. E. Van Doren Eds. (Plenum Press, 1979) p. 117.

[3] Pouget J. P., Ravy S. and Hennion B., Phase transitions 30 (1991) 5.

[4] Guinier A., X-ray diffraction in Crystals, Imperfect Crystals, and Amorphous Bodies (San Francisco: W. H. Freeman and company, 1963).

[5] a) Warren B. E., X-Ray diffraction (Addison-Wesley Publishing Company, 1969);

b) Warren B. E., Averbach B. L., Roberts B. W., J. Appl. Phys. 22 (1951) 1493.

[6] Cowley J. M., Acta Cryst. A 24 (1968) 557.

[7] CowLEY J. M., Diffraction physics (North-Holland Publishing Company, 1975).

[8] Shirley C. G. and Wilkins S., Phys. Rev. B 6 (1972) 1252.

[9] Welberry T. R., J. Appl. Cryst. 19 (1986) 382.

[10] Phillips T. E., Kistenmacher T. J., Bloch A. N. and Cowan D. O., J.C.S. Chem. Commun. (1976) 335.

[11] Yamaji K., Pouget J. P., Comes R. and Bechgaard K., J. Phys. Colloq. France 44 (1983) C31321.

[12] Bouveret Y. and Megtert S., J. Phys. France 50 (1989) 1649.

[13] See for example Sham L. J., in Ref. [2] p. 227 ;

ZuPPIROLI L., in Ref. [1] p. 437.

[14] RAVY S., POUGET J. P. and FrIEDEL J., in preparation.

[15] Levelut A. M., J. Phys. France 51 (1990) 1517. 